\title{
A Simple Method to Make Asymptotic Series of Feynman Diagrams Converge
}

\author{
Y. Meurice \\ Department of Physics and Astronomy, The University of Iowa, Iowa City, Iowa 52242, USA
}

\begin{abstract}
We show that for two non-trivial $\lambda \phi^{4}$ problems (the anharmonic oscillator and the LandauGinzburg hierarchical model), improved perturbative series can be obtained by cutting off the large field contributions. The modified series converge to values exponentially close to the exact ones. For $\lambda$ larger than some critical value, the method outperforms Padé's approximants and Borel summations. The method can also be used for series which are not Borel summable such as the double-well potential series. We show that semi-classical methods can be used to calculate the modified Feynman rules, estimate the error and optimize the field cutoff.
\end{abstract}

PACS: 11.10.-z, 11.15.Bt, 12.38.Cy, 31.15.Md

Perturbative series associated with Feynman diagrams are commonly used in particle physics, solid state physics, optics and chemistry 11. One remarkable success of this method is the prediction of the values of the anomalous magnetic moments of the electron and the muon with an incredible accuracy. Perturbative methods are also used to perform precision tests of the standard model of electro-weak and strong interactions [2]. Despite these successes, it has been known for a long time [3, 4 , that series calculated from Feynman diagrams are not convergent but asymptotic. In other words, the range of validity shrinks with the order. One can improve this situation by using Padé approximants [5], either on the original series or a Borel sum [6] of the series, if meaningful. However, even in the cases where the convergence of these alternate procedures can be proven, the convergence is very slow when the coupling is too large. In addition, for short series, it is difficult to estimate the error and to choose the best approximants.

In this Letter, we construct improved perturbative series which converge to values which are exponentially close to the exact ones. The error can be estimated analytically. The method can be applied on the lattice and in the continuum and works well when the methods mentioned above are inefficient or not applicable. The method is a perturbative version of recent numerical calculations performed for various $\lambda \phi^{4}$ models, namely the anharmonic oscillator [7] and the Landau-Ginzburg model in the hierarchical approximation [8]. In these calculations, we were led to introduce large $\phi$ cutoffs and realized that as $\lambda$ increases, the field cutoff can be decreased without affecting the accuracy of the result. The calculations presented here are perturbative series calculated with a large field cutoff. We only consider $\lambda \phi^{4}$ problems, however the procedure should extend to any kind of model where large field configurations are suppressed at positive coupling. This is in agreement with the general argument [9] linking the large field configurations to the impossibility of applying Lebesgue dominated convergence to the path integral expression of the perturbative series.

In order to give an idea about the efficiency of existing methods, we consider the well-known example of the ground state energy of the anharmonic oscillator [10]. The solid lines of Fig. 1 1 represent the number of significant digits obtained with perturbation theory for various $\lambda$. As the order increases, the approximate lines rotate clockwise while moving left, forming an approximate envelope. For a fixed coupling, there is an order of perturbation for which the error is minimized. On the other hand, the number of digits obtained with Padé approximants increases with the order. This is a consequence of Carleman's theorem which can be used to show 11] that diagonal sequences of Padé approximants converge to the ground state energy in an appropriately restricted domain of the complex plane. However, the convergence rate becomes slower as the coupling increases. This can be explained [11] from the fact that, when the coupling increases, a $[L / L]$ approximant tends to a constant while the energy increases like $\lambda^{1 / 3}$. If Padé approximants are used for the Borel transform instead of the series, one obtains results qualitatively similar which are discussed later.

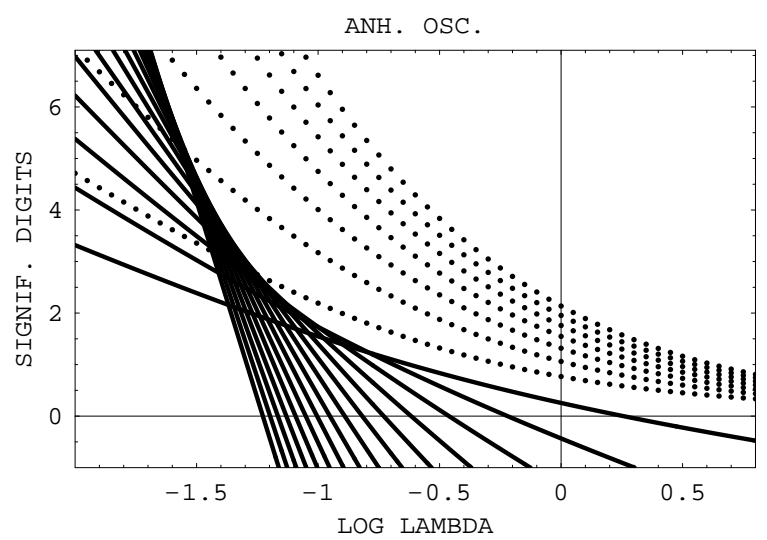

FIG. 1. Number of correct significant digits obtained with regular perturbation theory (solid lines) at order $1,2,3, \ldots$, 15 and with Padé approximants $[2 / 2],[3 / 3], \ldots . .[7 / 7]$ (dots) for the anharmonic oscillator, vs. $\log _{10} \lambda$. The various orders can be identified from the explanations in the text. In all the graphs, the logarithms are in base 10 .

The method that we propose provides a systematic and 
controllable improvement to regular perturbation and a better convergence than the Padé based methods in the right hand part of Fig. 11. It can also be used in cases where the Borel sum has singularities on the positive real axis. In particle physics, the experimental error bars of precision measurements are shrinking and higher order terms of perturbative series are being calculated. Firmly established discrepancy or agreement between theory and experiment provides valuable information regarding the laws of nature at shorter distances. A common practice 12] to estimate at which order, for a given coupling, we reach the envelope illustrated in Fig. 1 , is to determine when the ratio of successive contributions reaches one. This method works quite accurately for the three examples considered below. It seems reasonable to interpret ratios of successive contributions close to one as a signal that one needs to go beyond regular perturbative theory. We are getting close to this situation. For instance, the electro-weak corrections to $g_{\mu}-2$, give a contribution 113 of $151(4) \times 10^{-11}$ and in this calculation, the twoloop effects reduce the one-loop prediction by 35 percent. The total electroweak contribution is about one third of the discrepancy of $43(16) \times 10^{-10}$ found by the recent Brookhaven experiment [14]. The problem is more serious in the case of QCD corrections. For instance, in the calculation 15 of the hadronic width of the $Z^{0}$, the term of order $\alpha_{s}^{3}$ is more than 60 percent of the term of order $\alpha_{s}^{2}$ and contributes to one part in 1,000 to the total width.

We claim that introducing large field cutoffs leads to significantly improved perturbative series. An important reference to understand the general mechanism and to interpret the results presented below is the well studied [4.9] integral

$$
Z(\lambda)=\int_{-\infty}^{+\infty} d \phi \mathrm{e}^{-(1 / 2) \phi^{2}-\lambda \phi^{4}}
$$

If we expand $\mathrm{e}^{-\lambda \phi^{4}}$, the integrand for the order $p$ contribution is $\mathrm{e}^{-(1 / 2) \phi^{2}} \phi^{4 p} / p$ ! and has its maximum when $\phi^{2}=4 p$. On the other hand, the truncation of $\mathrm{e}^{-\lambda \phi^{4}}$ at order $p$ is accurate provided that $\lambda \phi^{4}<<p$. Requiring that the peak of the integrand for the $p$-th order term is within the range of values of $\phi$ for which the $p$ th order truncation provides an accurate approximation, yields the condition $\lambda<<(16 p)^{-1}$. One sees that the range of validity for $\lambda$ shrinks as one increases the order. We can avoid this problem by restricting the range of integration in Eq. (11) to $|\phi|<\phi_{\max }$. We call the truncated integral $Z\left(\lambda, \phi_{\max }\right)$. As the order increases, the peak of the integrand moves across $\phi_{\max }$ and the contribution is suppressed. It is easy to show that the coefficients of the modified series satisfy the bound $\left|a_{p}\right|<\sqrt{2 \pi} \phi_{\max }^{4 p} / p$ ! and the modified series defines an entire function. However, we are now constructing a perturbative series for a problem which is slightly different than the original one. This procedure is justified from the fact that the error is controlled by the inequality

$$
\left|Z(\lambda)-Z\left(\lambda, \phi_{\max }\right)\right|<2 \mathrm{e}^{-\lambda \phi_{\max }^{4}} \int_{\phi_{\max }}^{\infty} d \phi \mathrm{e}^{-(1 / 2) \phi^{2}} .
$$

We have applied large field cutoffs to the anharmonic oscillator with an Hamiltonian $H=p^{2} / 2+\phi^{2} / 2+\lambda \phi^{4}$. We use units such that the mass, the frequency and $\hbar$ are unity. The method of Ref. [7] was used to obtain a solution of the time-independent Schroedinger equation for an arbitrary value of the energy $E$. The eigenvalues are determined by using Sturm-Liouville theorem to monitor the "entrance" of the zeroes of the wave function in a region $0 \leq \phi \leq \phi_{\max }$ as $E$ increases. If $\phi_{\max }$ is large enough, one obtains excellent numerical values of $E_{n}$ for $n$ not too large by requiring that the $n+1$-th zero occurs exactly at $\phi_{\max }$. This numerical procedure can be converted into a perturbative expansion order by order in $\lambda$. By taking $\phi_{\max }$ large enough, namely 8 , we were able to reproduce accurately the first twenty terms of the series for the ground state calculated by Bender and $\mathrm{Wu}$ [10] without using diagrammatic techniques. We have also applied large field cutoffs to Dyson's hierarchical model, an approximation of lattice scalar field theory where the renormalization group transformation can be calculated numerically with great accuracy [8]. We have used a local Lagrangian density of the Landau-Ginzburg form $-A \phi^{2}-\lambda \phi^{4}$ such that when $\lambda=0$, the mass is unity. The field cutoff appears in the calculation of the Fourier transform of the local measure necessary for the numerical procedure. The free parameter (called $c / 4$ in Ref. [8]) appearing in the kinetic term was chosen in a such a way that a free massless field scales as it would for a nearest neighbor model in 3 dimensions.

We now present numerical results concerning the perturbative series for $Z\left(\lambda, \phi_{\max }\right)$, the ground state of the anharmonic oscillator and the zero-momentum two point function of the Landau-Ginzburg model. In Fig. 2, we compare the accuracy of regular perturbation theory with what is obtained with various field cutoffs. The curves for the modified series reach an asymptotic value on the left and drop on the right with the same slope as regular perturbation theory but with an intercept on the coupling axis shifted to the right. In between the two regimes, the curve reaches a maximum. As the order increases, the maximum moves up and right making the convergence apparent in this region. In all cases, the modified models provide an accuracy that goes far beyond the envelope of regular perturbation theory. We have then compared our results with those obtained with Padé approximants. For the clarity of the figure, we have limited ourselves to order 3 and 4 but similar situations are observed for higher orders. At each order, we have selected the best approximant for the series or its Borel sum. These sums are obtained by dividing the $l$-th coefficient by $\Gamma[l+1+b]$. We have followed the procedure of Ref. [6], except that at the end the inverse integral transform was performed numerically at fixed value of $\lambda$. We call this procedure the Padé-Borel method. 


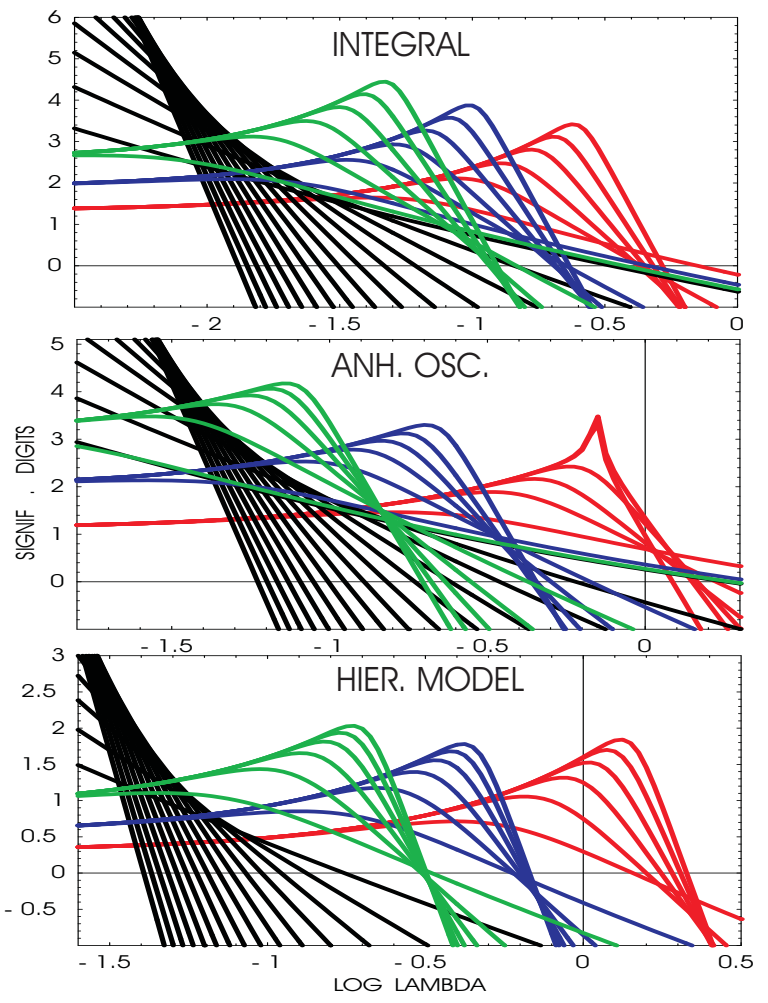

FIG. 2. Number of significant digits obtained with regular perturbation theory at order $1,3,5, \ldots ., 15$ (black) and with $\phi_{\max }=3$ (green), 2.5 (blue) and 2 (red), at order $1,3, \ldots, 11$ , as a function of $\lambda$, for the three quantities described in the text. Even orders have high cusps and are not displayed.

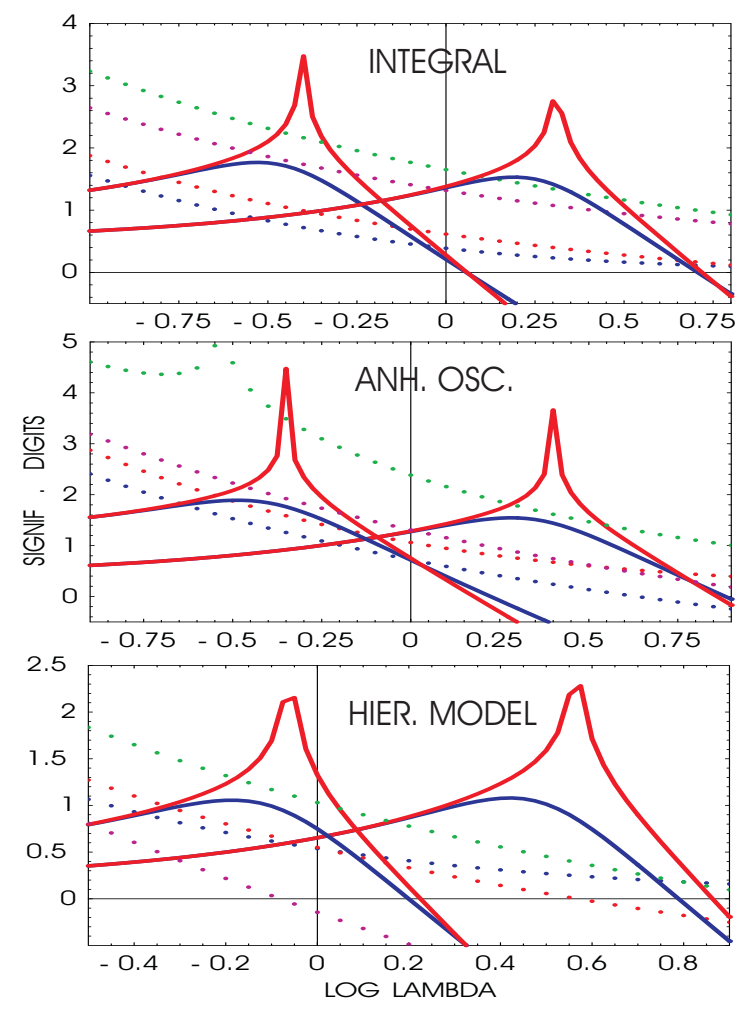

FIG. 3. Number of significant digits obtained with field cutoffs given in the text, at order 3 (blue line) and 4 (red line) compared to the best approximants for the regular series at order 3 (blue dots) and 4 (red dots) or the best results obtained with Padé-Borel method at order 3 (purple) and 4 (green).

The values of $b$ were adjusted to get the best possible result. At order 4 , the best approximant is $[2 / 2]$ in the 6 cases considered, but at order 3 , the situation is more complicated. In summary, we have used our knowledge of the exact result to get the best possible result for the methods to which we compare our results. Random choices of approximants or of the value of $b$ lead to significantly worse results. The results are shown in Fig. 3. We have used field cutoffs of 1.5 and 1 for the integral and 2 and 1.5 for the two other models. As the field cutoff decreases, the curve moves right as in Fig. 2. By comparing the three methods at the same order, we see that beyond a certain value of $\lambda$, the method used here outperforms the two methods based on Padé approximants within a certain range (which broadens when the coupling increases).

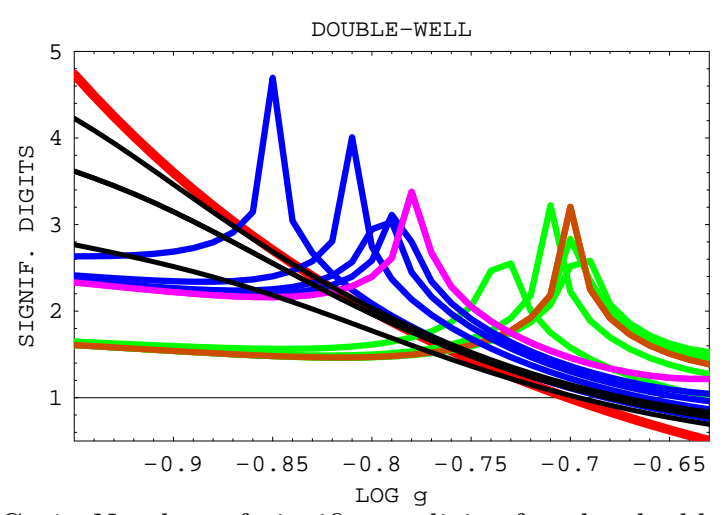

FIG. 4. Number of significant digits for the double-well at order 3 to 6 for regular perturbation (black) compared to series obtained with $y_{\min }=-3$ and $y_{\max }=3$ (blue) or $y_{\max }=2.5$ (green). As the order increases, the black curves reach the one-instanton contribution (red) over wider regions to the left while the two other sets reach the accuracy level obtained numerically for $y_{\max }=3$ (purple) or $y_{\max }=2.5$ (brown).

In all the examples considered above, the Borel sum has no singularity on the positive real axis. One can introduce such singularities by adding a cubic interaction with an appropriate coupling. However, for all examples worked out, this modification can be handled properly with the proposed method. This is due to the fact that as in the previous examples, the exponentials converge uniformly in a compact neighborhood of the origin, and it is legitimate to interchange the sum and the integral. We report here the case of the double-well potential in quantum mechanics as discussed in Ref. [16]. In shifted coordinates, the potential reads $(1 / 2) y^{2}-g y^{3}+\left(g^{2} / 2\right) y^{4}$. By imposing the vanishing of the wave function at $y= \pm 10$, 
we were able to reproduce all the significant digits of the first 10 coefficients for the ground state given in Table I of Ref. 16. This series is not Borel-summable. We have then constructed a modified series by imposing the wavefunction to vanish at $y_{\min }<0$ and its derivative to vanish at $y_{\max }>0$. If this prescription is used for numerical purposes, one obtains arbitrarily accurate results when $g=\left(1 / 2 y_{\max }\right)$ and $y_{\min }$ negative enough. The numerical results are shown in Fig. A for values of $g$ where the one-instanton contribution accounts for most of the discrepancy obtained with the regular series. The modified series converge rapidly to the numerical value obtained with the corresponding $y_{\min }$ and $y_{\max }$. It takes into account instanton effects and significantly improves the regular perturbative answer. Significant improvements have also been obtained for larger $g$ by decreasing $y_{\max }$.

The striking resemblance among the three models appearing in Figs. 2 and 3 suggests that, in general, the corrections due to the field cutoffs can be expressed as simple one-dimensional integrals. The perturbative expansion of the partition function of an arbitrary lattice scalar field theory with a large field cutoff can be obtained by writing the truncated integral at each site as the integral over the whole real axis minus the integral over $|\phi|>\phi_{\max }$. Regrouping the contributions with $0,1, \ldots$ large field contributions, we obtain the partition function

$$
\begin{array}{r}
Z[J]=C \mathrm{e}^{-\lambda \sum_{x}\left(\frac{\partial}{\partial J(x)}\right)^{4}} \mathrm{e}^{\frac{1}{2} \sum_{y, z} J(y) G(y, z) J(z)} \times \\
\left(1-A_{1} \sum_{y} \int_{\left|\phi_{y}\right|>\phi_{\max }} \mathrm{e}^{-A_{2}\left(\phi_{y}-\sum_{z} G(y, z) J_{z}\right)^{2}}+\ldots\right),
\end{array}
$$

with $A_{1}=(2 \pi G(0,0))^{-1 / 2}, A_{2}=(2 G(0,0))^{-1}, G(x, y)$ being the two-point function at $\lambda=0$ (with no field cutoff) and all quantities being written in lattice spacing units. The dots in Eq. (3) are calculable and exponentiate in the limit of a coarse lattice where the correlations among the sites are small. In general, Eq. (3) can be interpreted in terms of Feynman diagrams. A continuum version of this expression can be obtained by using a dilute-gas approximation for configurations with one "lump" of large values. We carried the detail of this calculation in the case of the anharmonic oscillator using the classical configuration $\phi_{\max } \mathrm{e}^{-\left|\tau-\tau_{0}\right|}$ and adapting the arguments of Ref. [17]. The result for the zero-th order correction to the ground state reads:

$$
\delta E_{0}^{(0)} \simeq 4 \pi^{-1 / 2} \phi_{\max }^{2} \int_{\phi_{\max }}^{+\infty} d \phi \mathrm{e}^{-\phi^{2}} .
$$

This prediction fits the numerical data over a wide range of $\phi_{\max }$. We can estimate the optimal value of $\phi_{\max }$ without knowing the numerical answer. The left part of the curves shown in Fig. 2 can be estimated by semi classical methods while the right part is given by the next order contribution. In the case of the anharmonic oscillator, using the classical configuration mentioned above, we obtain the error at order $n$ :

$$
\left|\delta E_{o}(\lambda)\right| \simeq \delta E_{o}^{(o)} \mathrm{e}^{-(1 / 2) \lambda \phi_{\max }^{4}}+\left|a_{n+1}\right| \lambda^{n+1}
$$

This approximate formula fits the data very well if $\phi_{\max }$ is not too small and allows a good estimate of the value of the coupling where the accuracy peaks.

The semi-classical calculation performed for the anharmonic oscillator can be extended to other scalar theories with exponentially decaying two-point functions and we expect an exponential control of the error for these models. In the case of lattice gauge theory, the integration over the fields is already reduced to a compact space. In the literature on lattice perturbation theory (with the exception of van Baal [18]), one usually replaces $\int d g$ by $\int_{-\infty}^{+\infty} d A_{\mu}^{i}$ since in the continuum limit the range becomes infinite. We claim that this lattice artifact can be used to obtain a smooth truncation of the perturbative series as in the scalar case. Different approximations need to be developed to solve the massless quadratic theory with a field cutoff. We expect that, as in the case of the double-well, this approach will lead to a quantitative understanding of the instanton effect.

This research was supported in part by the Department of Energy under Contract No. FG02-91ER40664.

[1] R. Feynman, Quantum Electrodynamics (Princeton University Press, Princeton, 1985).

[2] A. Sirlin, eConf C990809, 398 (2000).

[3] F. Dyson, Phys. Rev. 85, 32 (1952).

[4] J. C. Le Guillou and J. Zinn-Justin, Large-Order Behavior of Perturbation Theory (North Holland, Amsterdam, 1990) ands Refs. therein.

[5] G. Baker and P. Graves-Morris, Padé Approximants (Cambridge University Press, Cambridge, 1996).

[6] G. Baker, B. Nickel, M. Green, and D. Meiron, Phys. Rev. Lett. 36, 1351 (1976).

[7] B. Bacus, Y. Meurice, and A. Soemadi, J. Phys. A 28, L381 (1995).

[8] J. Godina, Y. Meurice, and M. Oktay, Phys. Rev. D 57, R6581 (1998) and D 59, 096002 (1999).

[9] S. Pernice and G. Oleaga, Phys. Rev. D 57, 1144 (1998).

[10] C. Bender and T. T. Wu, Phys. Rev. 184, 1231 (1969).

[11] J. Loeffel, A. Martin, B. Simon, and A. Wightman, Phys. Lett. B 30, 656 (1969).

[12] See e.g., J. Ellis, E. Gardi, M. Karliner, and M. Samuel, Phys. Lett. B366, 268 (1996).

[13] A. Czarnecki, B. Krause, and W. J. Marciano, Phys. Rev. Lett. 76, 3267 (1996).

[14] H. N. Brown et al., Phys. Rev. Lett. 86, 2227 (2001).

[15] S. A. Larin, T. van Ritbergen, and J. A. M. Vermaseren, Phys. Lett. B320, 159 (1994).

[16] E. Brezin, G. Parisi and J. Zinn-Justin, Phys. Rev. D 16, 408 (1977).

[17] S. Coleman, Aspects of Symmetry (Cambridge University Press, Cambridge, 1985).

[18] P. van Baal, Nucl. Phys. B 351, 183 (1991). 